\title{
EXACT BOUNDARY OBSERVABILITY FOR QUASILINEAR HYPERBOLIC SYSTEMS*
}

\author{
TATSIEN Li (DAQIAN Li) ${ }^{1}$
}

\begin{abstract}
By means of a direct and constructive method based on the theory of semi-global $C^{1}$ solution, the local exact boundary observability is established for one-dimensional first order quasilinear hyperbolic systems with general nonlinear boundary conditions. An implicit duality between the exact boundary controllability and the exact boundary observability is then shown in the quasilinear case.
\end{abstract}

Mathematics Subject Classification. 35B37, 93C20, 35L50, 93B07, 35R30

Received February 13, 2006. Revised April 2, 2007.

Published online January 30, 2008.

\section{INTRODUCTION AND MAIN RESUlTS}

Many studies have been done on the subject related to the exact boundary observability for linear hyperbolic systems (see [1-3,7,9,11-13], for instance). In this situation there is a duality between the controllability and the observability. The essential idea of the HUM suggested by J.-L. Lions in the study of controllability [7] is first to establish the corresponding observability inequality and then to get the controllability via the duality. For quasilinear hyperbolic systems, however, there is no such duality and the observability seems to be still open up to now.

In this paper we will establish the local exact boundary observability for the following one-dimensional first order quasilinear hyperbolic system

$$
\frac{\partial u}{\partial t}+A(u) \frac{\partial u}{\partial x}=F(u)
$$

where $u=\left(u_{1}, \ldots, u_{n}\right)^{T}$ is the unknown vector function of $(t, x), A(u)$ is an $n \times n$ matrix with suitably smooth entries $a_{i j}(u)(i, j=1, \ldots, n)$, and $F(u)=\left(f_{1}(u), \ldots, f_{n}(u)\right)^{T}$ is a suitably smooth vector function with

$$
F(0)=0
$$

By hyperbolicity, for any given $u$ on the domain under consideration, the matrix $A(u)$ possesses $n$ real eigenvalues

$$
\lambda_{1}(u), \ldots, \lambda_{n}(u)
$$

Keywords and phrases. Exact boundary observability, exact boundary controllability, semi-global $C^{1}$ solution, mixed initialboundary value problem, quasilinear hyperbolic system.

* Supported by the National Basic Research Program of China (973 Program) (2007CB814800).

1 School of Mathematical Sciences, Fudan University, Shanghai 200433, China; dqli@fudan.edu.cn 
and a complete set of left (resp. right) eigenvectors $l_{i}(u)=\left(l_{i 1}(u), \ldots, l_{i n}(u)\right)\left(\operatorname{resp} . r_{i}(u)=\left(r_{1 i}(u), \ldots, r_{n i}(u)\right)^{T}\right)$ :

$$
l_{i}(u) A(u)=\lambda_{i}(u) l_{i}(u) \quad\left(\text { resp. } A(u) r_{i}(u)=\lambda_{i}(u) r_{i}(u)\right) .
$$

Without loss of generality, we assume that

$$
l_{i}(u) r_{j}(u) \equiv \delta_{i j} \quad(i, j=1, \ldots, n)
$$

and

$$
r_{i}^{T}(u) r_{i}(u) \equiv 1 \quad(i=1, \ldots, n),
$$

where $\delta_{i j}$ denotes the Kronecker's symbol.

In what follows we suppose that there is no zero eigenvalue, namely, on the domain under consideration we have

$$
\lambda_{r}(u)<0<\lambda_{s}(u) \quad(r=1, \ldots, m ; s=m+1, \ldots, n) .
$$

In what follows the subscripts $r=1, \ldots, m$ (resp. $s=m+1, \ldots, n)$ are always used to correspond to the negative (resp. positive) eigenvalues.

Let

$$
v_{i}=l_{i}(u) u \quad(i=1, \ldots, n) .
$$

The boundary conditions are prescribed in the following most general form for the well-posedness:

$$
\begin{aligned}
& x=0: \quad v_{s}=G_{s}\left(t, v_{1}, \ldots, v_{m}\right)+H_{s}(t) \quad(s=m+1, \ldots, n), \\
& x=L: \quad v_{r}=G_{r}\left(t, v_{m+1}, \ldots, v_{n}\right)+H_{r}(t) \quad(r=1, \ldots, m),
\end{aligned}
$$

where $L$ is the length of the space interval $0 \leq x \leq L, H_{i}$ and $G_{i}(i=1, \ldots, n)$ are suitably smooth functions and

$$
G_{i}(t, 0, \ldots, 0) \equiv 0 \quad(i=1, \ldots, n) .
$$

Noting (1.2), $u=0$ is an equilibrium to the system (1.1).

Similarly to the study on the local exact boundary controllability $[5,6]$, we will use a direct and constructive method based on the theory of semi-global $C^{1}$ solution (see [4]) to get the local exact boundary observability in a neighbourhood of $u=0$. The main results are given in the following theorems.

Theorem 1.1 (two-side observation). Let

$$
T>L \max _{\substack{r=1, \ldots, m \\ s=m+1, \ldots, n}}\left(\frac{1}{\left|\lambda_{r}(0)\right|}, \frac{1}{\lambda_{s}(0)}\right) .
$$

Suppose that the $C^{1}[0, T]$ norm of $H(t)=\left(H_{1}(t), \ldots, H_{n}(t)\right)$ is suitably small. For any given initial condition

$$
t=0: \quad u=\varphi(x), \quad 0 \leq x \leq L
$$

such that $\|\varphi\|_{C^{1}[0, L]}$ is suitably small and the conditions of $C^{1}$ compatibility for the mixed initial-boundary value problem $(1,1),(1.13)$ and $(1.9)-(1.10)$ are satisfied at the points $(t, x)=(0,0)$ and $(0, L)$ respectively, if we have the observed values $v_{r}=\bar{v}_{r}(t)(r=1, \ldots, m)$ at $x=0$ and $v_{s}=\overline{\bar{v}}_{s}(t)(s=m+1, \ldots, n)$ at $x=L$ on the interval $[0, T]$, then the initial data $\varphi(x)$ can be uniquely determined and the following observability inequality holds:

$$
\|\varphi\|_{C^{1}[0, L]} \leq C\left(\sum_{r=1}^{m}\left\|\bar{v}_{r}\right\|_{C^{1}[0, T]}+\sum_{s=m+1}^{n}\left\|\overline{\bar{v}}_{s}\right\|_{C^{1}[0, T]}+\|H\|_{C^{1}[0, T]}\right),
$$

where $C$ is a positive constant. 
Remark 1.1. In Theorem 1.1 the observation is taken on both sides and the number of observed values is equal to $n$, the number of unknown functions.

Theorem 1.2 (two-side observation with less observed values). Suppose that the number of positive eigenvalues is less than that of negative ones:

$$
\bar{m} \triangleq n-m<m, \quad \text { i.e., } n<2 m \text {. }
$$

Suppose furthermore that in a neighbourhood of $u=0$, boundary condition (1.9) can be equivalently rewritten as

$$
x=0: \quad v_{\bar{r}}=\bar{G}_{\bar{r}}\left(t, v_{\bar{m}+1}, \ldots, v_{m}, v_{m+1}, \ldots, v_{n}\right)+\bar{H}_{\bar{r}}(t) \quad(\bar{r}=1, \ldots, \bar{m})
$$

with

then

$$
\bar{G}_{\bar{r}}(t, 0, \ldots, 0) \equiv 0 \quad(\bar{r}=1, \ldots, \bar{m}),
$$

$$
\overline{\bar{C}} \sum_{s=m+1}^{n}\left\|H_{s}\right\|_{C^{1}} \leq \sum_{\bar{r}=1}^{\bar{m}}\left\|\bar{H}_{\bar{r}}\right\|_{C^{1}} \leq \bar{C} \sum_{s=m+1}^{n}\left\|H_{s}\right\|_{C^{1}},
$$

where $\bar{C}$ and $\overline{\bar{C}}$ are positive constants. Let

$$
T>L\left(\max _{r=1, \ldots, m} \frac{1}{\left|\lambda_{r}(0)\right|}+\max _{s=m+1, \ldots, n} \frac{1}{\lambda_{s}(0)}\right) .
$$

Suppose finally that $\|H\|_{C^{1}[0, T]}$ is suitably small. For any given initial condition (1.13) with the same property as presented in Theorem 1.1, if we have the observed values $v_{\bar{s}}=\bar{v}_{\bar{s}}(t)(\bar{s}=\bar{m}+1, \ldots, m)$ at $x=0$ and $v_{s}=\overline{\bar{v}}_{s}(t)(s=m+1, \ldots, n)$ at $x=L$ on the interval $[0, T]$, then the initial data $\varphi(x)$ can be uniquely determined and the following observability inequality holds:

$$
\|\varphi\|_{C^{1}[0, L]} \leq C\left(\sum_{\bar{s}=\bar{m}+1}^{m}\left\|\bar{v}_{\bar{s}}\right\|_{C^{1}[0, T]}+\sum_{s=m+1}^{n}\left\|\overline{\bar{v}}_{s}\right\|_{C^{1}[0, T]}+\|H\|_{C^{1}[0, T]}\right),
$$

where $C$ is a positive constant.

Remark 1.2. Condition (1.16) in Theorem 1.2 and condition (1.21) in Theorem 1.3 below are imposed for guaranteeing the well-posedness of the corresponding backward mixed initial-boundary value problem. These conditions generalize the group condition given in Russell [8] for the special case that the problem is linear and the number of positive eigenvalues is equal to that of negative ones.

Remark 1.3. In Theorem 1.2, the observation is still taken on both sides, however, the number of observed values reduces to $(m-\bar{m})+\bar{m}=m$, the maximum value between the number of positive eigenvalues and the number of negative eigenvalues, and only $m-\bar{m}$ (instead of $m$ ) observed values are needed at $x=0$, namely, on the side with less boundary conditions.

Theorem 1.3 (one-side observation). Suppose that (1.19) hold and the number of positive eigenvalues is less than or equal to that of negative ones:

$$
\bar{m} \triangleq n-m \leq m, \quad \text { i.e., } n \leq 2 m \text {. }
$$

Suppose furthermore that in a neighbourhood of $u=0$, the boundary condition (1.10) implies

$$
x=L: \quad v_{s}=\bar{G}_{s}\left(t, v_{1}, \ldots, v_{\bar{m}}, v_{\bar{m}+1}, \ldots, v_{m}\right)+\bar{H}_{s}(t) \quad(s=m+1, \ldots, n)
$$

with

$$
\bar{G}_{s}(t, 0, \ldots, 0) \equiv 0 \quad(s=m+1, \ldots, n),
$$


then

$$
\sum_{s=m+1}^{n}\left\|\bar{H}_{s}\right\|_{C^{1}} \leq \bar{C} \sum_{r=1}^{m}\left\|H_{r}\right\|_{C^{1}}
$$

where $\bar{C}$ is a positive constant. Suppose finally that $\|H\|_{C^{1}[0, T]}$ is suitably small. For any given initial condition (1.13) with the same property as presented in Theorem 1.1, if we have the observed values $v_{r}=\bar{v}_{r}(r=$ $1, \ldots, m)$ at $x=0$ on the interval $[0, T]$, then the initial data $\varphi(x)$ can be uniquely determined and the following observability inequality holds:

$$
\|\varphi\|_{C^{1}[0, L]} \leq C\left(\sum_{r=1}^{m}\left\|\bar{v}_{r}\right\|_{C^{1}[0, T]}+\|H\|_{C^{1}[0, T]}\right),
$$

where $C$ is a positive constant.

Remark 1.4. In Theorem 1.3, the observation is taken only at $x=0$, namely, on the side with less boundary conditions, and the number of observed values is still equal to $m$, the maximum value between the number of positive eigenvalues and the number of negative eigenvalues as in Theorem 1.2. In particular, in the case that the number of positive eigenvalues is equal to that of negative ones: $n=2 m$, the observation can be taken on each side and the number of observed values is $m=\frac{n}{2}$.

Remark 1.5. When

$$
H_{i}(t) \equiv 0 \quad(i=1, \ldots, n)
$$

(1.14), (1.20) and (1.25) reduce to the corresponding observability inequalities with usual fashion, respectively.

The arrangement of this paper is as follows: in Section 2 we recall briefly the result on the semi-global $C^{1}$ solution. The proof of Theorems $1.1-1.3$ is given in Sections 3 and 4 respectively. Finally, we show in Section 5 that even in the quasilinear case there is still an implicit duality between the exact boundary controllability and the exact boundary observability.

\section{Semi-Global $C^{1}$ Solution}

Consider the mixed initial-boundary value problem for system (1.1) with the initial condition (1.13) and the boundary conditions (1.9)-(1.10).

By [4] (see also [10]), we have

Theorem 2.1. Suppose that on the domain under consideration, $\lambda_{i}(u), l_{i}(u), f_{i}(u), G_{i}(t,),. H_{i}(t)(i=$ $1, \ldots, n)$ and $\varphi(x)$ are all $C^{1}$ functions with respect to their arguments. Suppose furthermore that (1.2), (1.7) and (1.11) hold and the conditions of $C^{1}$ compatibility are satisfied at the points $(t, x)=(0,0)$ and $(0, L)$ respectively. For any preassigned and possibly quite large $T_{0}>0$, if the $C^{1}$ norms $\|\varphi\|_{C^{1}[0, L]}$ and $\|H\|_{C^{1}\left[0, T_{0}\right]}$ are suitably small (depending on $T_{0}$ ), then the mixed initial-boundary value problem (1.1), (1.13) and (1.9)-(1.10) admits a unique semi-global $C^{1}$ solution $u=u(t, x)$ with small $C^{1}$ norm on the domain $R\left(T_{0}\right)=\left\{(t, x) \mid 0 \leq t \leq T_{0}\right.$, $0 \leq x \leq L\}$. Moreover, under additional assumption that $\frac{\partial G_{i}}{\partial t}(t,).(i=1, \ldots, n)$ satisfy the local Lipschitz condition with respect to variables $v=\left(v_{1}, \ldots, v_{n}\right)$, we have

$$
\|u\|_{C^{1}\left[R\left(T_{0}\right)\right]} \leq C\left(\|\varphi\|_{C^{1}[0, L]}+\|H\|_{C^{1}\left[0, T_{0}\right]}\right),
$$

where $C$ is a positive constant possibly depending on $T_{0}$.

Corollary 2.1. Suppose that on the domain under consideration, $\lambda_{i}(u), l_{i}(u), f_{i}(u)(i=1, \ldots, n)$ and $\varphi(x)$ are all $C^{1}$ functions with respect to their arguments and (1.2) holds. If the $C^{1}$ norm $\|\varphi\|_{C^{1}[0, L]}$ is suitably small, then Cauchy problem (1.1) and (1.13) admits a unique global $C^{1}$ solution $u=u(t, x)$ on the whole maximum determinate domain and

where $C$ is a positive constant.

$$
\|u\|_{C^{1}} \leq C\|\varphi\|_{C^{1}[0, L]}
$$




\section{Proof of Theorem 1.1}

Since $\|\varphi\|_{C^{1}[0, L]}$ and $\|H\|_{C^{1}[0, T]}$ are small, by Theorem 2.1 the $C^{1}$ norms $\left\|\bar{v}_{r}\right\|_{C^{1}[0, T]}(r=1, \ldots, m)$ of the observed values $v_{r}=\bar{v}_{r}(t)(r=1, \ldots, m)$ at $x=0$ are also small. Using boundary condition (1.9), the values $\bar{v}_{s}(t)$ of $v_{s}(s=m+1, \ldots, n)$ at $x=0$ can be determined by

$$
\bar{v}_{s}(t)=G_{s}\left(t, \bar{v}_{1}(t), \ldots, \bar{v}_{m}(t)+H_{s}(t) \quad(s=m+1, \ldots, n) .\right.
$$

Noting (1.11), it is easy to see that

$$
\sum_{m+1}^{n}\left\|\bar{v}_{s}\right\|_{C^{1}[0, T]} \leq C\left(\sum_{r=1}^{m}\left\|\bar{v}_{r}\right\|_{C^{1}[0, T]}+\sum_{s=m+1}^{n}\left\|H_{s}\right\|_{C^{1}[0, T]}\right)
$$

here and hereafter $C$ denotes a positive constant. Then, noting (1.5) and (1.6), the value $\bar{u}(t)$ of the solution $u$ at $x=0$ satisfies

$$
\|\bar{u}\|_{C^{1}[0, T]} \leq C\left(\sum_{r=1}^{m}\left\|\bar{v}_{r}\right\|_{C^{1}[0, T]}+\sum_{s=m+1}^{n}\left\|H_{s}\right\|_{C^{1}[0, T]}\right) .
$$

Similarly, the value $\overline{\bar{u}}(t)$ of the solution $u$ at $x=L$ satisfies

$$
\|\overline{\bar{u}}\|_{C^{1}[0, T]} \leq C\left(\sum_{s=m+1}^{n}\left\|\overline{\bar{v}}_{s}\right\|_{C^{1}[0, T]}+\sum_{r=1}^{m}\left\|H_{r}\right\|_{C^{1}[0, T]}\right) .
$$

Since there is no zero eigenvalue (see (1.7)), we may change the status of $t$ and $x$. By Corollary 2.1, the rightward Cauchy problem for system (1.1) with the initial condition

$$
x=0: \quad u=\bar{u}(t), \quad 0 \leq t \leq T
$$

admits a unique $C^{1}$ solution $u=\tilde{u}(t, x)$ on the whole maximum determinate domain and

$$
\|\tilde{u}\|_{C^{1}} \leq C\left(\sum_{r=1}^{m}\left\|\bar{v}_{r}\right\|_{C^{1}[0, T]}+\sum_{s=m+1}^{n}\left\|H_{s}\right\|_{C^{1}[0, T]}\right) .
$$

Similarly, the leftward Cauchy problem for system (1.1) with the initial condition

$$
x=L: \quad u=\overline{\bar{u}}(t), \quad 0 \leq t \leq T
$$

admits a unique $C^{1}$ solution $u=\tilde{\tilde{u}}(t, x)$ on the whole maximum determinate domain and

$$
\|\tilde{\tilde{u}}\|_{C^{1}} \leq C\left(\sum_{s=m+1}^{n}\left\|\overline{\bar{v}}_{s}\right\|_{C^{1}[0, T]}+\sum_{r=1}^{m}\left\|H_{r}\right\|_{C^{1}[0, T]}\right)
$$

Obviously, both $u=\tilde{u}(t, x)$ and $u=\tilde{\tilde{u}}(t, x)$ are the restriction of the solution $u=u(t, x)$ to the original mixed problem on the corresponding domains respectively. 
Noting (1.12) and the smallness of the data, these two maximum determinate domains must intersect each other. Then, there exists $T_{0}\left(0<T_{0}<T\right)$ such that the value $\hat{u}(x)$ of the solution $u$ on $t=T_{0}$ can be determined by $u=\tilde{u}(t, x)$ and $u=\tilde{\tilde{u}}(t, x)$. Hence, noting (3.6) and (3.8), we have

$$
\|\hat{u}\|_{C^{1}[0, L]} \leq C\left(\sum_{r=1}^{m}\left\|\bar{v}_{r}\right\|_{C^{1}[0, T]}+\sum_{s=m+1}^{n}\left\|\overline{\bar{v}}_{s}\right\|_{C^{1}[0, T]}+\|H\|_{C^{1}[0, T]}\right) .
$$

We now solve the following backward mixed initial-boundary value problem for system (1.1):

$$
\begin{array}{ll}
t=T_{0}: \quad u=\hat{u}(x), & 0 \leq x \leq L, \\
x=0: & v_{r}=\bar{v}_{r}(t) \quad(r=1, \ldots, m), \\
x=L: & v_{s}=\overline{\bar{v}}_{s}(t) \quad(s=m+1, \ldots, n) .
\end{array}
$$

The conditions of $C^{1}$ compatibility at the points $(t, x)=\left(T_{0}, 0\right)$ and $\left(T_{0}, L\right)$ are evidently satisfied respectively. By Theorem 2.1 and noting (3.9), $u=u(t, x)$ as the $C^{1}$ solution to this mixed problem on the domain $R\left(T_{0}\right)=\left\{(t, x) \mid 0 \leq t \leq T_{0}, 0 \leq x \leq L\right\}$ verifies

$$
\|u\|_{C^{1}} \leq C\left(\sum_{r=1}^{m}\left\|\bar{v}_{r}\right\|_{C^{1}[0, T]}+\sum_{s=m+1}^{n}\left\|\overline{\bar{v}}_{s}\right\|_{C^{1}[0, T]}+\|H\|_{C^{1}[0, T]}\right) .
$$

In particular, noting (1.13), we get immediately the desired observability inequality (1.14).

Remark 3.1. In Theorem 1.1, the observability time given by (1.12) is sharp. It essentially means that two maximum determinate domains for the leftward and rightward Cauchy problems must intersect each other.

\section{Proofs of Theorems 1.2 And 1.3}

Proof of Theorem 1.2. By means of boundary condition (1.10), the value $\overline{\bar{u}}(t)$ of the solution $u$ at $x=L$ can be determined by the observed values $v_{s}=\overline{\bar{v}}_{s}(t)(s=m+1, \ldots, n)$ and (3.4) holds.

By Corollary 2.1, the leftward Cauchy problem for system (1.1) with the initial condition (3.7) admits a unique $C^{1}$ solution $u=\tilde{\tilde{u}}(t, x)$ on the whole maximum determinate domain and (3.8) holds.

Noting (1.19) and the smallness of the data, this maximum determinate domain must intersect the $t$-axis. Then, there exists $T_{0}\left(0<T_{0}<T\right)$ such that the value $\hat{u}(x)$ of the solution $u$ on $t=T_{0}$ can be determined by $u=\tilde{\tilde{u}}(t, x)$ and

$$
\|\hat{u}\|_{C^{1}[0, L]} \leq C\left(\sum_{s=m+1}^{n}\left\|\overline{\bar{v}}_{s}\right\|_{C^{1}[0, T]}+\sum_{r=1}^{m}\left\|H_{r}\right\|_{C^{1}[0, T]}\right) .
$$

We now solve the backward mixed initial-boundary value problem for system (1.1) with the initial condition (3.10), the boundary condition (3.12) on $x=L$, the boundary condition (1.16) on $x=0$ and

$$
x=0: \quad v_{\bar{s}}=\bar{v}_{\bar{s}}(t) \quad(\bar{s}=\bar{m}+1, \ldots, m),
$$

where $v_{\bar{s}}=\bar{v}_{\bar{s}}(t)(\bar{s}=\bar{m}+1, \ldots, m)$ are the observed values at $x=0$ on the interval $[0, T]$. By Theorem 2.1 and noting (1.18), $u=u(t, x)$ as the $C^{1}$ solution to this mixed problem on the domain $R\left(T_{0}\right)$ satisfies

$$
\|u\|_{C^{1}} \leq C\left(\sum_{\bar{s}=\bar{m}+1}^{m}\left\|\bar{v}_{\bar{s}}\right\|_{C^{1}[0, T]}+\sum_{s=m+1}^{n}\left\|\overline{\bar{v}}_{s}\right\|_{C^{1}[0, T]}+\|H\|_{C^{1}[0, T]}\right) .
$$

In particular, we get the desired observability inequality (1.20). 
Proof of Theorem 1.3. By means of boundary condition (1.9), the value $\bar{u}(t)$ of the solution $u$ at $x=0$ can be determined by the observed values $v_{r}=\bar{v}_{r}(t)(r=1, \ldots, m)$ and (3.3) holds.

The rightward Cauchy problem for system (1.1) with the initial condition (3.5) admits a unique $C^{1}$ solution $u=\tilde{u}(t, x)$ on the whole maximum determinate domain and (3.6) holds.

Noting (1.19) and the smallness of the data, this maximum determinate domain must intersect $x=L$. Then, there exists $T_{0}\left(0<T_{0}<T\right)$ such that the value $\hat{u}(x)$ of the solution $u$ on $t=T_{0}$ can be determined by $u=\tilde{u}(t, x)$ and

$$
\|\hat{u}\|_{C^{1}[0, L]} \leq C\left(\sum_{r=1}^{m}\left\|\bar{v}_{r}\right\|_{C^{1}[0, T]}+\sum_{s=m+1}^{m}\left\|H_{s}\right\|_{C^{1}[0, T]}\right) .
$$

We now solve the backward mixed initial-boundary value problem for system (1.1) with the initial condition (3.10), the boundary condition (3.11) on $x=0$ and the boundary condition (1.22) on $x=L$. By Theorem 2.1 and noting (1.24), $u=u(t, x)$ as the $C^{1}$ solution to this mixed problem on the domain $R\left(T_{0}\right)$ satisfies

$$
\|u\|_{C^{1}} \leq C\left(\sum_{r=1}^{m}\left\|\bar{v}_{r}\right\|_{C^{1}[0, T]}+\|H\|_{C^{1}[0, T]}\right) .
$$

In particular, we get the desired observability inequality (1.25).

Remark 4.1. In Theorems 1.2 and 1.3, the observability time given by (1.19) is sharp. It essentially means that the maximum determinate domain for the leftward (resp. rightward) Cauchy problem much intersect $x=0$ (resp. $x=L)$.

\section{5. "Duality" BetWeEn CONTROLlability AND OBSERVABILITY}

Comparing Theorems 1.1 and 1.3 with the results in $[5,6,10]$ on the controllability, we may find an implicit duality between the exact boundary controllability and the exact boundary observability even in the quasilinear hyperbolic case.

In two-side case, we have:

(a) The controllability time is equal to the observability time (see (1.12)).

(b) The restriction on the controllability time essentially means that two maximum determinate domains for the forward and backward Cauchy problems respectively never intersect each other, while, the restriction on the observability time essentially means that two maximum determinate domains for the leftward and rightward Cauchy problems respectively must intersect each other.

(c) The procedure of resolution for getting the controllability is first to solve the forward and backward mixed problems and then to solve the leftward and rightward mixed problems, while, the procedure of resolution for getting the observability is first to solve the leftward and rightward Cauchy problems and then to solve the backward mixed problem.

(d) The number of controls is equal to $n$, the number of observed values.

In one-side case, we have:

(a) The controllability time is still equal to the observability time (see (1.19)).

(b) The restriction on the controllability time essentially means that two maximum determinate domains for the forward and backward one-side mixed problems respectively never intersect each other, while, the restriction on the observability time essentially means that the maximum determinate domain for the rightward Cauchy problem must intersect $x=L$.

(c) The procedure of resolution for getting the controllability is first to solve the forward and backward mixed problems and then to solve the rightward mixed problem, while, the procedure of resolution for getting the observability is first to solve the rightward Cauchy problem and then to solve the backward mixed problem. 
(d) Both the number of controls and the number of observed values are equal to the maximum value between the number of positive eigenvalues and that of negative ones.

(e) The control is taken on $x=L$, namely, on the side with more boundary conditions, while, the observation is taken on $x=0$, namely, on the side with less boundary conditions.

\section{REFERENCES}

[1] F. Alabau and V. Komornik, Observabilité, contrôlabilité et stabilisation frontière du système d'élasticité linéaire. C. R. Acad. Sci. Paris Sér. I Math. 324 (1997) 519-524.

[2] C. Bardos, G. Lebeau and R. Rauch, Sharp efficient conditions for the observation, control and stabilization of wave from the boundary. SIAM J. Control Optim. 30 (1992) 1024-1065.

[3] I. Lasiecka, R. Triggiani and P. Yao, Inverse/observability estimates for second-order hyperbolic equations with variable coefficients. J. Math. Anal. Appl. 235 (1999) 13-57.

[4] T. Li and Y. Jin, Semi-global $C^{1}$ solution to the mixed initial-boundary value problem for quasilinear hyperbolic systems. Chin. Ann. Math. 22B (2001) 325-336.

[5] T. Li and B. Rao, Local exact boundary controllability for a class of quasilinear hyperbolic systems. Chin. Ann. Math. 23B (2002) 209-218.

[6] T. Li and B. Rao, Exact boundary controllability for quasilinear hyperbolic systems. SIAM J. Control Optim. 41 (2003) $1748-1755$.

[7] J.-L. Lions, Contrôlabilité Exacte, Perturbations et Stabilisation de Systèmes Distribués, Tome I: Contrôlabilité Exacte, RMA 8. Masson (1988).

[8] D.L. Russell, Controllability and stabilizability theory for linear partial differential equations: recent progress and open questions. SIAM Rev. 20 (1978) 639-739.

[9] I. Trooshin and M. Yamamoto, Identification problem for a one-dimensional vibrating system. Math. Meth. Appl. Sci. 28 (2005) 2037-2059.

[10] Z. Wang, Exact controllability for nonautonomous first order quasilinear hyperbolic systems. Chin. Ann. Math. 27B (2006) 643-656.

[11] P. Yao, On the observability inequalities for exact controllability of wave equations with variable coefficients. SIAM J. Control Optim. 37 (1999) 1568-1599.

[12] E. Zuazua, Boundary observability for the space-discretization of the 1-D wave equation. C. R. Acad. Sci. Paris Sér. I Math. 326 (1998) 713-718.

[13] E. Zuazua, Boundary observability for the finite-difference space semi-discretizations of the $2-D$ wave equation in the square. J. Math. Pures Appl. 78 (1999) 523-563. 